\title{
Analysis of the fecal microbiota of fast- and slow-growing rainbow trout (Oncorhynchus mykiss)
}

Pratima Chapagain ${ }^{1}$, Brock Arivett ${ }^{1,2}$, Beth M. Cleveland ${ }^{3}$, Donald M. Walker ${ }^{1}$ and Mohamed Salem ${ }^{1,4^{*}}$ (D)

\begin{abstract}
Background: Diverse microbial communities colonizing the intestine of fish contribute to their growth, digestion, nutrition, and immune function. We hypothesized that fecal samples representing the gut microbiota of rainbow trout could be associated with differential growth rates observed in fish breeding programs. If true, harnessing the functionality of this microbiota can improve the profitability of aquaculture. The first objective of this study was to test this hypothesis if gut microbiota is associated with fish growth rate (body weight). Four full-sibling families were stocked in the same tank and fed an identical diet. Two fast-growing and two slow-growing fish were selected from each family for $16 \mathrm{~S}$ rRNA microbiota profiling.

Microbiota diversity varies with different DNA extraction methods. The second objective of this study was to compare the effects of five commonly used DNA extraction methods on the microbiota profiling and to determine the most appropriate extraction method for this study. These methods were Promega-Maxwell, Phenol-chloroform, MO-BIO, Qiagen-Blood/Tissue, and Qiagen-Stool. Methods were compared according to DNA integrity, cost, feasibility and inter-sample variation based on non-metric multidimensional scaling ordination (nMDS) clusters.

Results: Differences in DNA extraction methods resulted in significant variation in the identification of bacteria that compose the gut microbiota. Promega-Maxwell had the lowest inter-sample variation and was therefore used for the subsequent analyses. Beta diversity of the bacterial communities showed significant variation between breeding families but not between the fast- and slow-growing fish. However, an indicator analysis determined that cellulose, amylose degrading and amino acid fermenting bacteria (Clostridium, Leptotrichia, and Peptostreptococcus) are indicator taxa of the fast-growing fish. In contrary, pathogenic bacteria (Corynebacterium and Paeniclostridium) were identified as indicator taxa for the slow-growing fish.

Conclusion: DNA extraction methodology should be carefully considered for accurate profiling of the gut microbiota. Although the microbiota was not significantly different between the fast- and slow-growing fish groups, some bacterial taxa with functional implications were indicative of fish growth rate. Further studies are warranted to explore how bacteria are transmitted and potential usage of the indicator bacteria of fast-growing fish for development of probiotics that may improve fish health and growth.
\end{abstract}

Keywords: Aquaculture, Trout, Gut, Microbiota, DNA-isolation, Breeding

\footnotetext{
* Correspondence: mosalem@umd.edu

'Department of Biology and Molecular Biosciences Program, Middle

Tennessee State University, Murfreesboro, TN 37132, USA

${ }^{4}$ Department of Animal and Avian Sciences, University of Maryland, College

Park, MD 20742, USA

Full list of author information is available at the end of the article
}

(c) The Author(s). 2019 Open Access This article is distributed under the terms of the Creative Commons Attribution 4.0 International License (http://creativecommons.org/licenses/by/4.0/), which permits unrestricted use, distribution, and reproduction in any medium, provided you give appropriate credit to the original author(s) and the source, provide a link to the Creative Commons license, and indicate if changes were made. The Creative Commons Public Domain Dedication waiver (http://creativecommons.org/publicdomain/zero/1.0/) applies to the data made available in this article, unless otherwise stated. 


\section{Introduction}

The efficiency and profitability of industrial aquaculture depend in part on the growth rate of farmed fishes. Growth in farmed fishes is a complex process that is directly dependent on host genetics, food quality and availability, and environmental conditions [1]. Selective breeding is one strategy that can be used to improve important phenotypic traits and help in understanding the genetic architecture and the role of molecular factors causing genetic variation among different fish [2]. Family-based selection procedures have been undertaken by the United States Department of Agriculture (USDA), National Center for Cool and Cold-Water Aquaculture (NCCCWA) to improve growth rate, fillet quality and disease resistance of rainbow trout. A growth-selected line was developed starting in 2002, and since then yielded a genetic gain of approximately $10 \%$ in improved growth performance per generation [3].

Microorganisms may also contribute to the productivity of farmed fishes. Microorganisms making up the fish microbiota reside on the fish skin, gills, and gastrointestinal tract and likely play a crucial role in the growth rate, metabolism, and immunity of the fish host $[4,5]$. While host genetics has a profound role in determining the gut microbiome of humans and other mammals, it is not well studied in fish [6-9]. On the other hand, feed and water in which fish are reared have vital roles in shaping the gut microbiome. For example, plant and animal-based meal can widely alter the composition of the host microbiota since fish acquire their microbiota from the first-feed they eat [10-12]. Sharp et al. reported that microbiota of the marine species can be directly inherited from ancestors and passed from generation to generation [13]. The gut, in particular, features a diverse microbiota contributing to the weight gain, immune development, pathogen inhibition, and various metabolic activities of the hosts [14]. Resident gut microbes are beneficial for hosts either by inhibiting pathogenic bacteria with dedicated toxins or by secreting enzymes that breakdown indigestible polysaccharides in host gut to simple monosaccharides and short-chain fatty acids [15]. Gut microbes can supply compounds such as vitamin B and $\mathrm{K}$ to host which may improve the host energy metabolism [16].

An accurate census of bacteria from fish may allow investigation of the positive effects of the microbiota. However, profiling of the gut microbiome is directly influenced by many factors including the experimental design, sample collection, and processing. DNA extraction is particularly important since microbiome analysis requires adequate quality and quantity of DNA isolated for an accurate representation of the host-microbiome [17]. Many protocols have been commercialized for DNA extraction and previous reports demonstrate that microbiome diversity varies with different DNA extraction methods [18]. It is difficult to determine the most appropriate extraction method for the downstream microbiome analysis of a particular species. Each method has its own merits and drawbacks; for example, standardized kits are typically designed for ease of use and efficiency, but a more labor-intensive method such as Phenol-chloroform extraction, despite its risk of inconsistency or contamination, can potentially produce a higher yield with better quality if performed by an experienced researcher.

In this study, we investigated how the gut microbiota of rainbow trout correlates with differential growth rates. Therefore, one objective of this research was to characterize the gut microbiota of rainbow trout using high-throughput DNA sequencing. In order to achieve this objective, we considered the effect that DNA extraction methodologies play in the characterization of different microbial communities in the gut of rainbow trout. The specific objectives of our study were to determine differences in community structure of the gut microbiota between fast- and slow-growing rainbow trout and to determine if genetics plays a role in determining the gut microbiota profile. Our results highlight differences of the gut microbiota between fish family and the bacterial taxa indicative of fast- and slow-growing rainbow trout.

\section{Results}

\section{Comparison of different DNA extraction methods}

To test if profiling of the gut microbiota is directly influenced by the DNA extraction method, three replicate pools of the fish fecal samples were sequenced and analyzed using five different extraction methods. Within non-metric dimensional scaling ordination plots, the three-replicate samples extracted with Promega clustered tightly, whereas, replicate samples of the four other extraction methods were relatively more heterogeneous (Fig. 1). PERMANOVA confirmed that the microbial population differs on using different DNA extraction method $\left(\mathrm{F}_{4,13}=2.4234, p<0.05, \mathrm{R}^{2}=51 \%\right)$.

To further investigate the effects of DNA extraction methodology on microbiota profiling, three different methods were chosen for microbiota sequencing from the individual (non-pooled) biological replicate fecal samples of all available fish in the study. PERMANOVA results confirmed the significant effect of extraction technique on predicting microbial communities (Fig. 2 a; $\left.F_{2,42}=10.467, p<0.05, R^{2}=34 \%\right)$. Comparative analysis of the three extraction methods revealed that Phenolchloroform had the highest OTU richness with 649 OTUs. A total of 119 OTUs overlapped between all three DNA isolation methods (Fig. 2b). Comparing the abundance of the Gram-positive and Gram-negative bacteria, it was clear that the abundance of the Grampositive is higher than that of the Gram-negative in all 


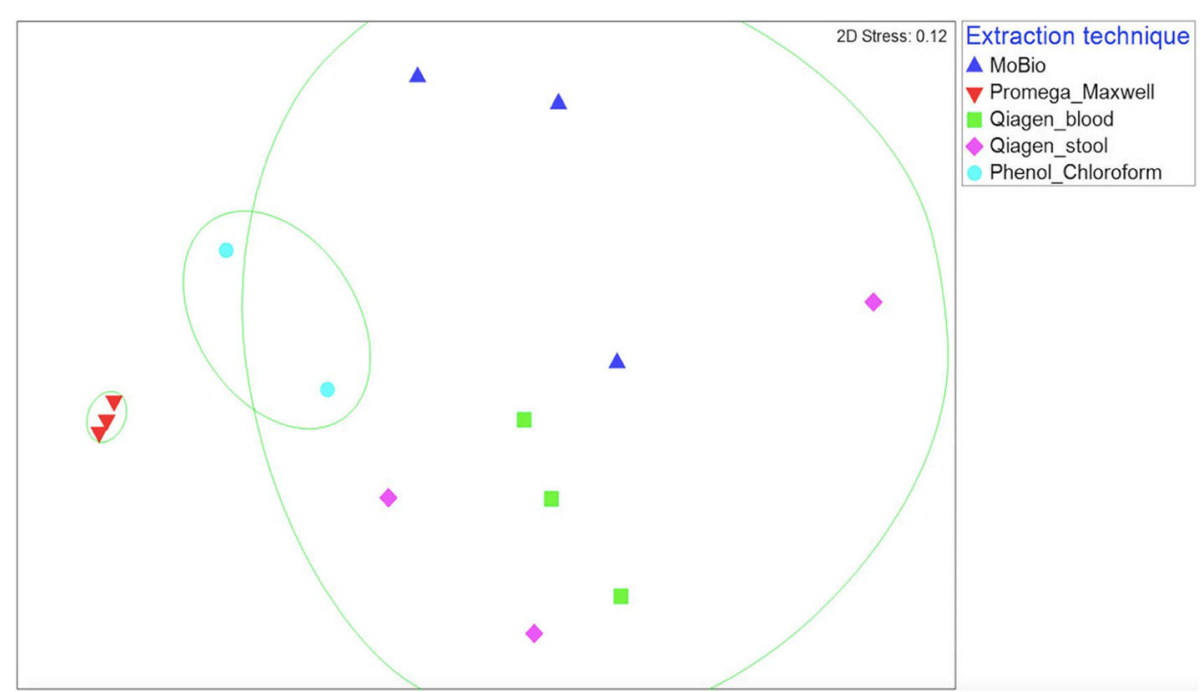

Fig. 1 nMDS representation of three replicate pooled samples using 5 different extraction methods (stress value $=0.12$ ). Each extraction method is significantly different $(p<0.05)$. SIMPROF analysis tested for significant distinct clusters. One of the phenol-chloroform samples did not pass the QC and was excluded from the analysis
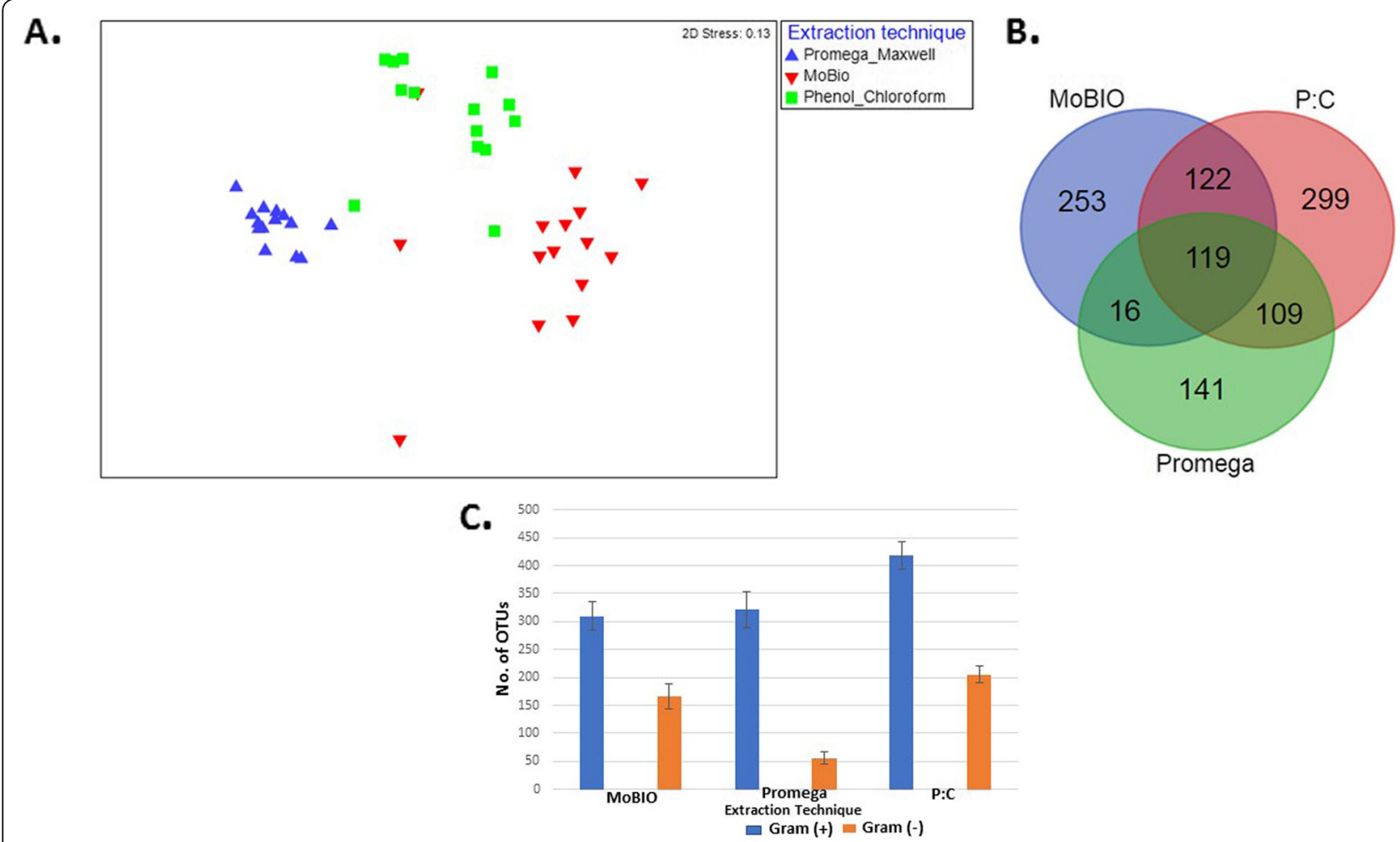

Fig. 2 a) nMDS representation of the fecal samples using three different extraction methods. Samples were clustered on the basis of Bray-Curtis distance matrices (stress value $=0.13$ ). b) Venn Diagram depicting the common and unique OTUs in three different extraction methods, P:C indicates phenol-chloroform c) Abundance of Gram-positive and Gram-negative bacteria on rainbow trout gut using three different extraction methods. The error bar indicates the standard deviation 
three DNA extraction techniques (Fig. 2c) with the Promega kit being the highest. The SIMPROF test for statistically significant cluster and it showed that the Promega method had $95 \%$ similarity within the individual samples forming the tightest cluster $(p<0.05)$.

Beside heterogeneity and abundance biases, other factors including yield, integrity, time durations for sample processing, the amount of hazardous waste liberated were also considered during extraction comparison. Phenol-chloroform gave the highest yield, but it is tedious, time-consuming, requires individual handling and released more hazardous waste whereas, Promega is a semi-automated method, easy to perform in large-scale production, and showed the least inter-sample variation among the replicate samples, results in release of least hazardous waste as shown in (Table 1). We decided to choose Promega for our downstream analysis of the fecal microbiota.

\section{Mean weight difference between fast and slow-growing fish}

The mean weight of the fast-growing fish was $2123.9 \pm$ $105.57 \mathrm{~g}$, whereas, the mean weight of the slow-growing fish was $988.6 \pm 297.65 \mathrm{~g}$. The mass of the fast-growing fish was significantly greater than that of the slowgrowing fish when compared using one-way MannWhitney $\mathrm{U}$ test $(p<0.05)$ as shown in Fig. 3.

\section{Gut microbiota analysis of fast- and slow-growing fish}

Our analysis of microbial diversity based on alpha diversity in the fast-growing and slow-growing fish fecal samples using inverse Simpson indices indicated no significant differences between fast and slow-growing fish ( $p>0.05$, data not shown). Moreover, both nMDS ordination and PERMANOVA results indicated that the microbial communities did not significantly differ between the fish of different growth rates ( $p>0.05$, Fig. 4a). Both fast- and slow-growing fish possessed unique sets of OTUs and overlapping taxa (Fig. 4b). However, an indicator analysis predicted that 10 OTUs were found as indicative of the growth rate (Table $2, p<0.05$ ). All fastgrowing indicator taxa belonged to phylum Firmicutes, including genera Clostridium, Sellimonas, Leptotrichia,
Tepidimicrobium, Peptostreptococcus and Lachnospiraceae_unclassified whereas, the slow-growing indicator taxa belonged to phylum Actinobacteria and Firmicutes with genera Corynebacterium and Paeniclostridium (Table 2).

In addition, PERMANOVA results indicated differences in the microbiota among the fish families $\left(\mathrm{F}_{3,13}=\right.$ 2.1673, $p<0.05, \quad \mathrm{R}^{2}=39 \%$ ) (Fig. 4c). The Vennrepresentation depicted 106 OTUs shared among all the families with family 2 having the most unique OTUs (Fig. 4d). An indicator analysis of each fish family predicted that six OTUs belonging to phylum Actinobacteria and Firmicutes including genera Truperella, Kocuria, Lactobacillus, Lactococcus were identified as indicative of family 1 . Three OTUs belonging to phylum Fusobacteria, Firmicutes including genera Fusobacterium and Peptostreptococcus were indicator taxa for family 2. And one OTUs belonging to phylum Proteobacteria including genus Pseudomonas was indicator taxa for family 4 (Table $3, p<0.05)$. The overall taxa information of the fecal samples has been included in Additional file 1.

Because the Phenol-chloroform yielded higher OTUs, despite the higher intersample variation among the replicates, as a curiosity, we ran the nMDS ordination and PERMANOVA analyses using the Phenol-chloroform extraction method. The results also indicated no significant differences among the growth rate $(\mathrm{p}<0.05)$ of fish with significant differences among the families $(\mathrm{p}<0.05)$ and alpha diversity analysis using inverse Simpson index also showed insignificant results $(p>0.05)$. These results resemble those obtained by the Promega extraction method.

\section{Discussion}

In this study, the DNA extraction methodology comparison was performed to optimize the extraction methodology and apply this to the comparison of fast- and slow-growing fish gut microbiota. Five different extraction techniques, including bead beating and semi-automated methods, were examined. The effects of the DNA extraction methods were assessed on the basis of the DNA quantity, quality and the inter-sample variation in microbial communities between replicates. The concentration and the quality of the DNA

Table 1 Comparison of five different DNA extraction methods for microbiota analysis on the basis of cost, concentration, and the time duration for sample processing

\begin{tabular}{lllllllcc}
\hline Extraction Kit & Manufacturer & Principle & Bead Beating & Concentration (ng/ $\mathrm{ll})$ & A260/230 & Cost per sample & $\begin{array}{l}\text { Time } \\
\text { duration }\end{array}$ & $\begin{array}{l}\text { Hazardous } \\
\text { waste }\end{array}$ \\
\hline Power Soil & MoBio & Manual & Yes & $6.49 \pm 9.09$ & $1.78 \pm 0.18$ & $\$ 6.48$ & $6 \mathrm{~h}$ & Moderate \\
Maxwell & Promega & Automated & Yes & $28.76 \pm 12.44$ & $1.72 \pm 0.17$ & $\$ 7.40$ & $1.5 \mathrm{~h}$ & Least \\
Phenol:Chloroform & Sigma & Manual & No & $257.1 \pm 285.0$ & $1.73 \pm 0.08$ & $\$ 4.50$ & 2 days & High \\
Qiagen_Stool & Qiagen & Manual & No & $25.1 \pm 10.07$ & $1.92 \pm 0.16$ & $\$ 5.60$ & $5 \mathrm{~h}$ & Less \\
Qiagen_Blood/Tissue & Qiagen & Manual & No & $35.2 \pm 2.7$ & $1.72 \pm 0.01$ & $\$ 4.20$ & $5 \mathrm{~h}$ & Less \\
\hline
\end{tabular}




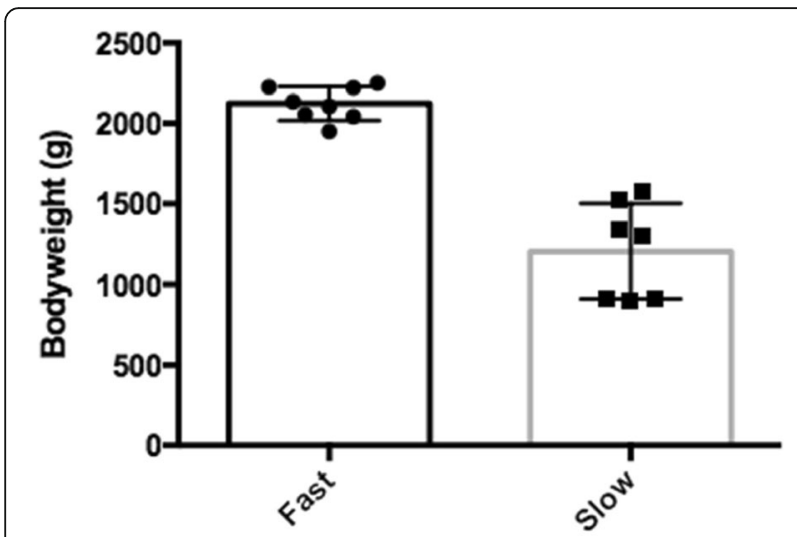

Fig. 3 Significant difference in the mean weight of the fast-growing versus slow-growing fish used in the study. The statistical significance of the rank body mass between the two groups was tested by a one-way Mann-Whitney $U$ test $(p<0.05)$. The error bars indicate standard deviation varied significantly between the DNA extraction techniques. The MOBIO, Qiagen Blood/Tissue and Qiagen Stool gave relatively low yield, whereas Promega Maxwell kit that uses automated method resulted in a higher yield in comparison to the other kits which is consistent with previous reports [19]. In comparison, Phenol-chloroform, being a robust method, uses a stringent lysis step and produced the highest DNA yield and highest microbial diversity. This is likely due to the Phenol-chloroform method being able to effectively lyse the cell walls of both the Gram-positive and Gram-negative bacteria. However, the Phenolchloroform method resulted in higher inter-sample variation, is the most labor-intensive, and produces more hazardous waste when compared to the Promega method. It has been proven that the bead-beating methods result in the identification of greater microbial diversity than nonbeating methods [20]. MOBIO method, involves bead beating to physically lyse cell wall of bacteria, increased the number of the microbial species identified but showed relatively high inter-sample variation among replicates. Promega Maxwell, a semi-automated method, also includes bead-beating steps, however, yielded a higher abundance of Gram-positive bacteria, perhaps, due to addition of

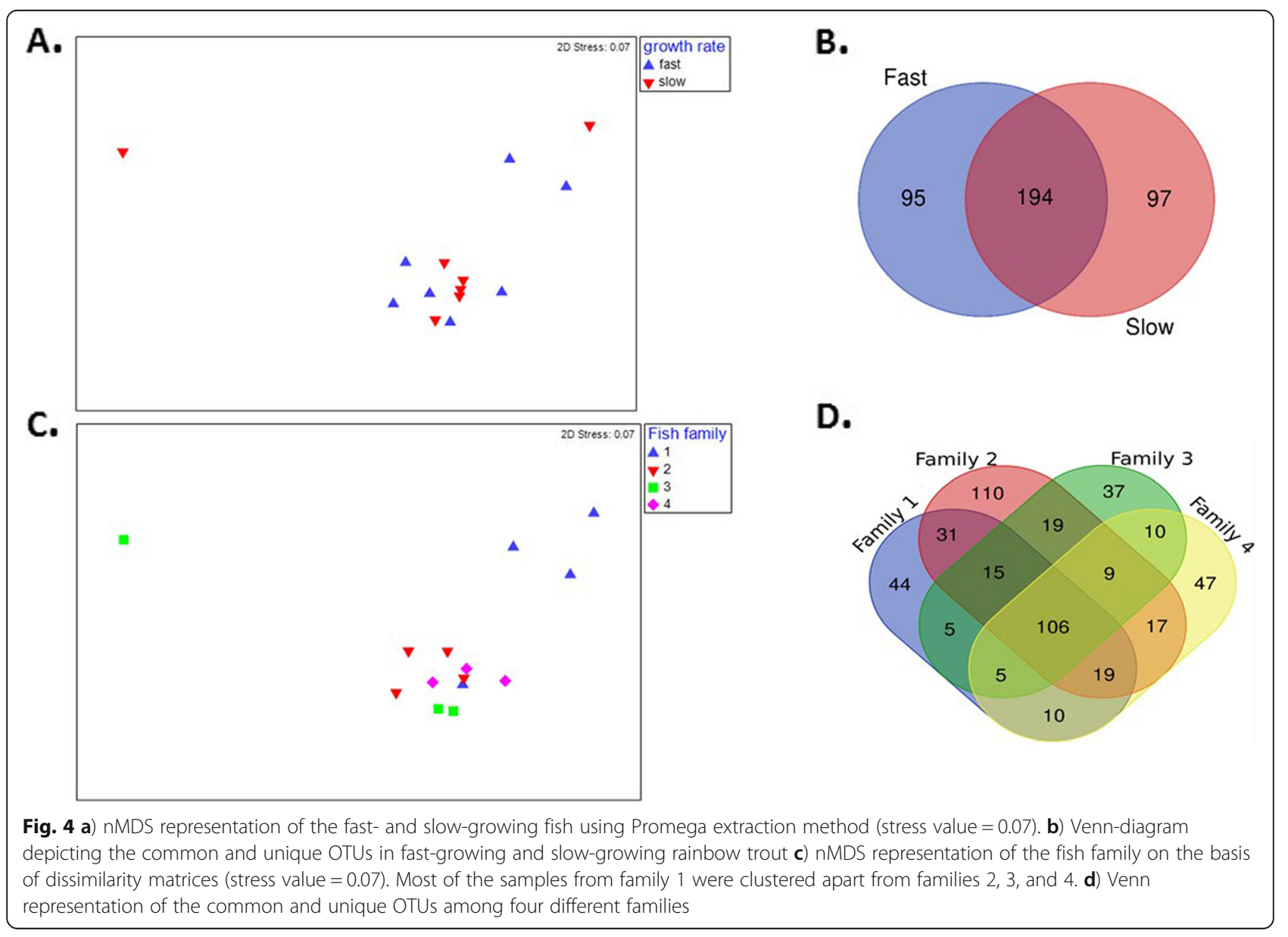


Table $\mathbf{2}$ Indicator analysis of the taxa for growth rate using Mothur

\begin{tabular}{|c|c|c|c|c|c|c|c|c|}
\hline Growth & Phylum & Class & Order & Family & Genus & Abundance & $\begin{array}{l}\text { Indicator } \\
\text { Value }\end{array}$ & $P$-value \\
\hline \multirow[t]{8}{*}{ Fast } & Firmicutes & Clostridia & Clostridiales & Clostridiaceae_1 & Clostridium_sensu_stricto_1 & 1589 & 86 & $<0.001$ \\
\hline & Firmicutes & Clostridia & Clostridiales & Lachnospiraceae & Sellimonas & 1265 & 66 & 0.03 \\
\hline & Fusobacteria & Fusobacteriia & Fusobacteriales & Leptotrichiaceae & Leptotrichia & 940 & 75 & 0.03 \\
\hline & Firmicutes & Clostridia & Clostridiales & Clostridiaceae_1 & Clostridium_sensu_stricto_18 & 761 & 78 & 0.04 \\
\hline & Firmicutes & Clostridia & Clostridiales & Family_XI & Tepidimicrobium & 456 & 77 & 0.03 \\
\hline & Firmicutes & Bacilli & Bacillales & Planococcaceae & Planococcaceae_unclassified & 388 & 79 & 0.01 \\
\hline & Firmicutes & Clostridia & Clostridiales & Lachnospiraceae & Lachnospiraceae_unclassified & 357 & 78 & 0.02 \\
\hline & Firmicutes & Clostridia & Clostridiales & Peptostreptococcaceae & Peptostreptococcus & 139 & 80 & 0.01 \\
\hline \multirow[t]{2}{*}{ Slow } & Actinobacteria & Actinobacteria & Corynebacteriales & Corynebacteriaceae & Corynebacterium_1 & 10,033 & 74.07 & 0.01 \\
\hline & Firmicutes & Clostridia & Clostridiales & Peptostreptococcaceae & Paeniclostridium & 958 & 65 & 0.04 \\
\hline
\end{tabular}

$p \leq 0.05$ indicates the significant taxa to act as indicator of the fast-growing or slow-growing fish

lysozyme enzymes, which induces lysis of the Grampositive bacterial cell wall. The Promega method showed the least inter-sample variation among technical replicates. Similar is the case with Qiagen-stool, Qiagen-Blood/Tissue kits since both methods gave sufficient yield and integrity but resulted in higher inter-sample variation among replicates.

We found that specific taxa were indicators of the fish growth rate and fish breeding family. The indicator taxa associated with slow growth rate seem to be harmful/ pathogenic bacteria, whereas the indicator taxa of fastgrowing fish seem to have a mutually beneficial relationship with the host. Corynebacterium and Paeniclostridium which are known pathogens [21] were more prevalent in slow-growing fish. The toxins produced by these bacteria cause swelling and abdominal discomfort due to fluid accumulation and sometimes also lead to the development of circumscribed lesions and lethargic behavior [22]. Families Lachnospiraceae, Leptotrichiaceae, Planococcaceae, and Peptostreptococcaceae belonging to the phylum Firmicutes were indicator taxa for the fast-growing fish in this study. Firmicutes impact fatty acid absorption and lipid metabolism, thus expected to affect body weight in the host [23-25]. A study done in Zebrafish explained the contribution of Firmicutes in stimulating the host metabolism and increasing the bioavailability of fatty acids by modifying bile salts [26]. Bacteria belonging to class Lachnospiraceae reside in the digestive tract, produce butyric acid, aid in amino acid fermentation, protein digestion, absorption of fatty acids, were associated with weight gain and prevention of different diseases due to microbial and host epithelial cell growth $[27,28]$. On the other hand, bacteria like Sellimonas, Clostridium, Peptostreptococcus in fast-growing fish can take part in fermentation of different amino acids, lactates and sugars [29]. Clostridium is more likely to produce cellulase enzyme and result in degradation of the cellulolytic fibers. The most widely prevalent and statistically significant indicator taxa of the fast-growing fish, Peptostreptococcus and Clostridium, are more likely to be involved in amino acid fermentation that ultimately leads to amino acid absorption in host gut. Leptorichia, the most abundant taxa in the gut of all the fastgrowing fish are cellulose-degrading bacteria; therefore, amylase and cellulase activities are expected to be more prominent in the host inhabiting these bacteria [30]. Similarly, the class Enterobacteriaceae was found to be a significantly abundant taxonomical class in most of the fast-growing fish. E. coli belonging to class Enterobacteriaceae has proven to be associated with weight gain in human infants [31].

Although most of the microbiota were shared among the fish families, some unique taxa were characteristic for each family, which suggests that genetics is a contributing factor affecting the gut microbiota. Unique taxa for fish family 1 included Trueperiolla, Kocuria, Lactobacillus, Lactococcus, and Propionibacteriaceae. Kocuria has been reported to induce the protective immune system in rainbow trout by inhibiting pathogenic bacteria like Vibrio [32]. Lactobacillus has been found to inhibit the pathogens and, therefore, used as preservatives for food storage since they can induce the barrier function in the host epithelium against pathogens [33]. Also, bacteria belonging to family Propionibacteriaceae produce microbial metabolites such as short-chain fatty acids during glucose fermentation [34]. The bacteria belonging to this family also produce enzymes for fatty acid degradation that may help in the breakdown of food and produce valuable nutrients and energy [29, 35-37]. Similarly, Fusobacterium, an indicator taxon of fish family 2 produces butyrate which supplies energy, enhances mucus production and induces anti-inflammatory properties in the host [38]. Fish family 3 showed a higher abundance of phylum Bacteroidales with unclassified 
Table 3 Indicator analysis of the taxa for fish families using Mothur

\begin{tabular}{|c|c|c|c|c|c|c|c|c|}
\hline $\begin{array}{l}\text { Fish } \\
\text { Family }\end{array}$ & Phylum & Class & Order & Family & Genus & Abundance & $\begin{array}{l}\text { Indicator } \\
\text { value }\end{array}$ & $p$-value \\
\hline \multirow[t]{7}{*}{1} & Actinobacteria & Actinobacteria & Actinomycetales & Actinomycetaceae & Trueperella & 9007 & 53.15 & 0.02 \\
\hline & Actinobacteria & Actinobacteria & Micrococcales & Micrococcaceae & Kocuria & 5226 & 57.95 & 0.007 \\
\hline & Firmicutes & Bacilli & Lactobacillales & Lactobacillaceae & Lactobacillus & 1233 & 68.78 & 0.02 \\
\hline & Firmicutes & Clostridia & Clostridiales & Ruminococcaceae & $\begin{array}{l}\text { Ruminococcaceae_ } \\
\text { UCG-014 }\end{array}$ & 615 & 65.49 & 0.03 \\
\hline & Firmicutes & Bacilli & Lactobacillales & Streptococcaceae & Lactococcus & 589 & 73.38 & 0.015 \\
\hline & Actinobacteria & Actinobacteria & Propionibacteriales & Propionibacteriaceae & Propionibacteriaceae & 134 & 52.7 & 0.02 \\
\hline & Fusobacteria & Fusobacteriia & Fusobacteriales & Fusobacteriaceae & Fusobacterium & 1048 & 61.53 & 0.03 \\
\hline \multirow[t]{3}{*}{2} & Firmicutes & Clostridia & Clostridiales & Peptostreptococcaceae & Peptostreptococcus & 110 & 65.57 & 0.02 \\
\hline & Firmicutes & Clostridia & Clostridiales & Family_XIII & $\begin{array}{l}\text { Family_XIII__ } \\
\text { unclassified }\end{array}$ & 86 & 63.15 & 0.03 \\
\hline & Bacteroidetes & Bacteroidia & Bacteroidales & $\begin{array}{l}\text { Bacteroidales_ } \\
\text { unclassified }\end{array}$ & $\begin{array}{l}\text { Bacteroidales_ } \\
\text { unclassified }\end{array}$ & 12,125 & 99.49 & 0.04 \\
\hline \multirow[t]{2}{*}{3} & Firmicutes & Bacilli & Bacillales & Paenibacillaceae & Paenibacillus & 360 & 70.31 & 0.019 \\
\hline & Actinobacteria & Coriobacteriia & Coriobacteriales & Atopobiaceae & $\begin{array}{l}\text { Atopobiaceae_- } \\
\text { unclassified }\end{array}$ & 196 & 63.414 & 0.01 \\
\hline 4 & Proteobacteria & Gammaproteobacteria & Pseudomonadales & Pseudomonadaceae & Pseudomonas & 5265 & 76.19 & 0.01 \\
\hline
\end{tabular}

$p \leq 0.05$ indicates the significant indicator taxa for each fish family

family and genus. Bacteriodetes belonging to this phylum produces inhibitory substances like bacteriocin which initiates pathogenic bacterial cell lysis or growth inhibition [35]. Pseudomonas, an indicator taxon of family 4 has been identified as the gut microbiota that aid in digestion [10]. Differences in microbiota among the families suggest that host genetics may create a genetic background that promotes the specific selection of microbiota from the environment. However, it should also be acknowledged that early periods of development, before fish comingled for the grow-out period, occurred in different tanks specific to each family. Although all four tanks were positioned sequentially, utilized the same water source (inlets came originated from the same pipe), and consumed identical feed, it is unknown if the microbial communities within each tank differed and, if so, how they could have persisted through the subsequent 12-month grow-out period. It is also unknown if there is vertical microbiota transmission from the parents to progeny or if maternal fecal contamination of eggs during manual egg stripping contributes to the offspring microbiota. Further research is needed to validate familial differences and determine the contribution of genetic and environmental factors to development of the gut microbiota.

\section{Conclusion}

This study showed that DNA extraction methodology should be taken into account for accurate profiling of the gut microbiome. Some bacterial taxa were found to be significantly different between fish families, perhaps due to host genetics, unique early rearing environments, or vertical microbiota transmission. Although population-level microbiota differences were not found to be significantly associated with the fish growth rate, several indicator taxa were determined in the fast- and slow-growing fish. For future studies, some of these taxa can be investigated for potential use as probiotics to improve the gut microbiota of rainbow trout. Overall, our study investigated the gutpassing microbiota using fecal samples, which may not represent the mucosal microbiota.

\section{Methods}

\section{Fish population}

Fecal samples were collected from 15 fish representing four different genetic families. The parents of these families originated from a growth-selected line at NCCCWA (year class 2014) that was previously described [3, 39]. Fish families were produced and reared at NCCCWA until $\sim 18$ months post-hatch. Briefly, full-sibling families were produced from single-sire $\times$ single-dam mating events. All sires were siblings from a single-family while dams exhibited low relatedness (coefficient of relatedness $<0.16)$. Eggs were reared in spring water, and water temperatures were manipulated between approximately $7-13^{\circ} \mathrm{C}$ to synchronize hatch times. Each family was reared separately from hatch through approximately $20 \mathrm{~g}$ (7 months post-hatch) when 15 fish per family were uniquely tagged by inserting a passive integrated transponder (Avid Identification Systems Inc., Norco, CA) into the peritoneal cavity. Tagged fish were comingled for the remainder of the grow-out period. Fish were fed 
a commercial fishmeal-based diet (42\% protein, $16 \%$ fat, Ziegler Bros Inc., Gardners, PA) using automatic feeders (Arvotec, Huutokoski, Finland). Feed was provided at or just below satiation for the entire grow-out period. This study includes four families with high variance in adult body weight. From each family, four fish were selected, two that were considered fast-growing $(>1952 \mathrm{~g})$ and two that were slow-growing $(<1572 \mathrm{~g})$. Of the 16 fish selected for sampling, one slow-growing fish from family two exhibited morphological signs of disease during sample collection and was excluded from analysis, reducing the total number of samples to 15 .

\section{Sample collection}

To characterize the gut microbiota, samples were collected from fish feces. For fecal sampling, fish were anesthetized with MS-222 (tricane methane sulfonate) at a concentration of $150 \mathrm{mg} \mathrm{m} / \mathrm{L}$ (Tricaine-S, Western Chemical, Ferndale, WA) and then manually stripped to collect the fecal samples in sterile Eppendorf tubes (Eppendorf, Hauppauge, NY). All samples were stored at $-80^{\circ} \mathrm{C}$ until DNA extraction. At the end of the experiment, fish were euthanized with an overdose of MS-222 at a concentration of $300 \mathrm{mg} / \mathrm{L}$.

\section{DNA isolation and sequencing}

For comparison of extraction methods, fecal samples from 8 fast-growing and 7 slow-growing fish were pooled together and DNA extraction was done in triplicate using five different extraction methods including PowerSoil ${ }^{\circ}$ DNA Isolation Kit (MO BIO Laboratories, Inc., West Carlsbad, CA), Promega Maxwell DNA Isolation Kit (Promega Corporation, Madison, WI), Qiagen Blood/Tissue, Qiagen Stool (Qiagen, Germantown, MD) and Phenolchloroform (Phenol: Chloroform 5:1, SIGMA) extraction method [40]. The individual biological replicates DNA samples extracted using the MOBIO, Promega, and Phenol-chloroform methods were used for the analysis of the gut microbiota of fast-growing versus slow-growing trout. More detail of the DNA extraction methods is provided in Additional file 2 and steps of experimental design using pooled and unpooled samples have been included in Fig. 5. After extraction, DNA concentration was measured using Qubit (Qubit fluorometer, v3.11) (Invitrogen, Carlsbad, CA) and DNA was visualized by gel electrophoresis. All DNA extractions were stored at $-80^{\circ} \mathrm{C}$ until library preparation.

Before library preparation, concentrations of all DNA samples were normalized to $2 \mathrm{ng} / \mu \mathrm{L}$ for PCR amplification using a Qubit fluorometer. The primers $515 \mathrm{~F}$ and 926R (Integrated DNA Technologies) (EMP; http://www. earthmicrobiome.org/emp-standard-protocols/16s/) were used to target the $16 \mathrm{~S}$ rRNA marker gene using polymerase chain reaction (PCR). The final PCR reaction consisted of $5 \mu \mathrm{L}$ buffer, $1.5 \mu \mathrm{L} 50 \mathrm{mM} \mathrm{MgCl}, 2 \mu \mathrm{L} 10$ $\mathrm{mM}$ dNTP, $0.2 \mu \mathrm{L}$ Taq polymerase, $3 \mu \mathrm{L} \mathrm{Kb}$ extender, $1 \mu \mathrm{L} 10 \mu \mathrm{M}$ primer, $5 \mu \mathrm{L}$ DNA template and $7.3 \mu \mathrm{L}$ nuclease-free water. PCR amplification and sample indexing (a total of 67 samples multiplexed) was performed [41]. The amplification conditions were $94^{\circ} \mathrm{C}$ for $45 \mathrm{~s}, 50^{\circ} \mathrm{C}$ for $60 \mathrm{~s}, 72^{\circ} \mathrm{C}$ for $90 \mathrm{~s}$ for 35 cycles. Amplification was preceded by a 10 -min preheating step at $94{ }^{\circ} \mathrm{C}$ and followed by a 10 -min elongation step at $72{ }^{\circ} \mathrm{C}$. Amplification of each sample was performed in triplicate and combined to a final volume of $75 \mu \mathrm{L}$. The indexed samples were then normalized $(240 \mathrm{ng} /$ reaction) and pooled for sample purification purposes. The pooled amplicon was purified using Promega PCR purification kit (Promega Corporation, Madison, WI) and visualized on a $1.5 \%$ agarose gel stained with ethidium bromide. A DNA fragment of the amplicon for each sample was excised from the DNA gel with a clean, sharp scalpel and collected in nuclease-free sterile tubes. QIAquick gelextraction kit was used to purify DNA from the resulting gel slice (Qiagen, Germantown, MD) according to the manufacturer's recommendation. The concentration of the gel-extracted library was assessed with a Qubit fluorometer (Invitrogen, Carlsbard, CA) and fragment size was determined using an Agilent 2100 Bioanalyzer (Agilent, Santa Clara, California). Final qPCR-based quantification of the library was done using a KAPPA quantification kit (Roche, Pleasanton, CA). Sequencing was done using $250 \mathrm{bp}$ paired-end sequencing using a 300 cycle V2 reagent cartridge on an Illumina Miseq flow cell (Illumina, Inc., San Diego, CA) according to the manufacturer's instructions (Miseq System Guide) [42]. The output file was demultiplexed and converted to fastq on the Illumina MiSeq (Illumina, Inc., San Diego, CA).

\section{Bioinformatics analyses}

During sequencing, the adaptor trimming option was selected to remove the adaptors from the sequences. Samples were demultiplexed prior to using Mothur on the basis of Illumina Miseq instructions and total 8,500,662 paired-end raw sequences were obtained from Miseq Software (version 2.6.2.3). Sequencing data were analyzed using Mothur (v.1.40.2, www.mothur.org) according to the Mothur Illumina Miseq standard operating procedure (SOP) [43, 44] with several modifications. After forming contigs, the total number of sequences were $3,972,613$ the median length $(371 \mathrm{bp})$ of the sequences was determined. Sequences with ambiguous base pairs were removed by using the screen.seqs command, which ultimately reduced the sequences to 3,340 , 066. The split.abund command was used to keep sequences with more than two reads [45]. Since we were sequencing V4-V5 region, we customized our reference 


\section{DNA isolation and sequencing}

A.

\author{
Pooled fecal samples \\ from fast- and slow- \\ growing fish
}

B.

Fecal samples from 8
fast-growing and 7
slow-growing fish

(unpooled)
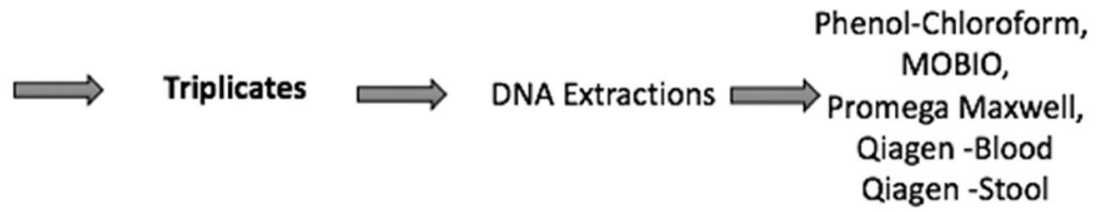

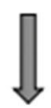

DNA

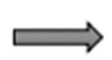

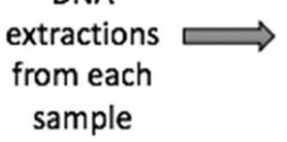

Promega Maxwell MOBIO

Phenol-Chloroform
Library Preparation

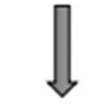

Sequencing

Fig. 5 Experimental design for DNA isolation and sequencing. a) DNA extraction comparison using pooled fecal samples from all fast- and slowgrowing fish. Three pooled fecal samples from all fast and slow-growing fish were subjected to five different DNA extraction comparisons. b) Analysis of fecal sample (unpooled) from 8 fast and 7 slow-growing fish to study the microbial assemblages

alignment using primer for V4-V5 region (http://blog. mothur.org/2016/07/07/Customization-for-your-region/), sequences were then trimmed on the basis of alignment start and end using pcr.seqs command. Filter.seqs command was used to filter the sequences with $\mathrm{QC}$ value $>25$ and 3,314,628 sequences were then aligned to the SILVA v123 database and sequences that failed to align, or classified as Archaea, chloroplast, eukaryotic mitochondrial, or unknown sequences, were excluded from the analysis. Sequences detected by UCHIME as chimeric were removed from the analysis. The remaining sequences $(3,150,919)$ were clustered using VSEARCH [46] at a threshold of > 97\% sequence similarity. The remove.rare command was used to remove operational taxonomic units (OTUs) with abundance less than ten reads $[47,48]$. Two samples (one fast-growing extracted using Promega Maxwell method and one slow-growing fish extracted using Phenolchloroform method) were excluded from the analysis because sequences in these samples did not pass the quality control and filtering steps. The parameters and the commands used to analyze the data are included in Additional file 3.

\section{Statistical analysis}

To study the effect of DNA extraction methods on microbial community profiling, Bray-Curtis distances were compared and nMDS ordination was used for visualization using Primer 7 (version 7.0.13) for windows ((PRIMER-E Ltd., Plymouth, UK). To test for a significant effect of extraction method, we used Permutational Multivariate Analysis of Variance (PERMANOVA) on the basis of Bray-Curtis dissimilarity matrices by considering extraction technique as a fixed effect and using type III sum of squares and unrestricted permutation of data with 999 permutations. SIMPROF (Similarity Profile) was performed to test the inter-sample variation on the replicate samples with a significant cut off value of 0.5 ( $95 \%$ similarity). Similarly, Beta diversity of fast-growing and slow-growing samples were calculated using Bray-Cutis dissimilarity matrices representing pairwise (sample to sample) distances to test the variation among fast and slow-growing fish. Non-metric multidimensional scaling ordination (nMDS) was used to explore the microbial communities in the fastgrowing and slow-growing fish by considering the dissimilarity distance matrices among the samples. A oneway PERMANOVA was used to determine if the microbial assemblages differ with growth rate or fish breeding family, both considered as fixed effects. Moreover, alpha diversity was evaluated by comparing inverse Simpson diversity matrices for each group i.e. fish growth rate and fish families using $R$ ( $R$ version 3.5.2).

To determine the microbial assemblages that are characteristics to the two growth rates and four families, an indicator species analysis was done in Mothur using indicator command $[25,49]$. Taxa with indicator value greater than 40 and a $(p<0.05)$ were considered as significant indicators of fish growth rate or a breeding family [49]. All data files for the bioinformatics and statistical analyses are included in Additional files 3, 4, 5, 6, 7, 8 and 9_b.

The statistical significance of the rank body mass between the two groups was tested by a one-way MannWhitney $\mathrm{U}$ test with an alpha of $\mathrm{p}<0.05$ (Prism, GraphPad Software, Inc., La Jolla, CA). 


\section{Supplementary information}

Supplementary information accompanies this paper at https://doi.org/10. 1186/s12864-019-6175-2

Additional file 1. Taxa information of trout fecal samples. Taxonomy information of fecal samples using Promega Maxwell DNA extraction kit.

Additional file 2. DNA extraction protocol. DNA extraction Protocols of three different extraction techniques: PowerSoil ${ }^{\oplus}$ DNA Isolation Kit - MO BIO, Phenol-Chloroform and Promega Maxwell 16 FFPE Plus LEV DNA Purification Kit.

Additional file 3. Mothur SOP. Mothur Standard Operating procedure for microbiota profiling.

Additional file 4. Mothur Analysis log file. Logfile containing Mothur commands and results during Mothur analysis. Result of each step can be tracked in this file.

Additional file 5. Metadata file for fast and slow, Extraction technique Detailed information regarding the fast and slow-growing fish samples (weight, length, sex, condition factor).

Additional file 6. a and 6 b. Mothur shared file. a) Fast- and slowgrowing fish fecal sample analysis shared file Extraction technique shared file. The data in the shared file contains the relative abundance of OTUs in multiple samples. This file can be used for further analysis, e.g. alpha and beta diversity measurements using different packages ( $R$, Primer)

Additional file 7. Beta diversity analysis result file of the fast- and slowgrowing fish using PRIMER. PRIMER was used for multivariate analysis. PERMANOVA results for fast and slow-growing fish analysis and PERMANOVA results for fish families.

Additional file $\mathbf{8}$. Beta diversity analysis result file of extraction technique using PRIME. PERMANOVA result for DNA extraction comparison.

Additional file 9. _a and 9_b. Mothur taxonomy file. a) Fast- and slowgrowing fecal analysis taxonomy file Extraction techniques taxonomy file. Data in taxonomy file contains the taxa information of OTUs that matched with the Silva database.

\section{Abbreviations}

nMDS: non-metric multidimensional scaling; OTUs: Operational Taxonomic units; PERMANOVA: Permutational Multivariate Analysis of Variance; rRNA: Ribosomal RNA

\section{Acknowledgments}

The authors acknowledge technical and animal caretaking contributions from Josh Kretzer and Kyle Jenkins. Mention or use of trade names does not represent endorsement by the USDA. The USDA is an equal opportunity provider and employer. We would like to thank Dr. Mary Farone for providing reagents and lab facilities during this project.

\section{Authors' contributions}

Conceived and designed the experiments: PC, BA, BC, MS. Performed the experiments: PC, BA, BC, DW, MS. Analyzed the data: PC, BA, DW, MS. Wrote the paper: PC, DW, MS. All authors read and approved the final manuscript.

\section{Funding}

No funding was obtained for this study.

\section{Availability of data and materials}

All data are provided in additional files.

\section{Ethics approval and consent to participate}

This study, including the husbandry practices, analysis and euthanasia methods of the rainbow trout was submitted to and approved by the Animal Care and Use Committee of the United States Department of Agriculture, National Center for Cool and Cold Water Aquaculture (IACUC approval \#098).

\section{Consent for publication}

Not applicable.

\section{Competing interests}

Mohamed Salem is a member of the editorial board of BMC Genomics. Otherwise, the authors declare that they have no competing interests.

\section{Author details}

${ }^{1}$ Department of Biology and Molecular Biosciences Program, Middle Tennessee State University, Murfreesboro, TN 37132, USA. ${ }^{2}$ Department of Chemistry, Middle Tennessee State University, Murfreesboro, TN 37132, USA. ${ }^{3}$ National Center for Cool and Cold-Water Aquaculture, ARS-USDA, Kearneysville, WV 25430, USA. ${ }^{4}$ Department of Animal and Avian Sciences, University of Maryland, College Park, MD 20742, USA.

Received: 1 August 2019 Accepted: 2 October 2019

Published online: 29 October 2019

References

1. Hamre J, Johnsen E, Hamre K: A new model for simulating growth in fish In.: Pubmed.gov; 2014 Jan 30.

2. Gjedrem T. Selection and breeding programs in aquaculture. New York: Springer; 2008.

3. Leeds TD, Vallejo RL, Weber GM, Pena DG, Silverstein JS. Response to five generations of selection for growth performance traits in rainbow trout (Oncorhynchus mykiss). Aquaculture. 2016;465:341-51.

4. Kau AL, Aherm PP, Griffin NW, Goodman AL, Gordon Jl. Human nutrition, the gut microbiome, and immune system: envisioning the future. In: PMC. 2011 Jun;15.

5. Merrifield DL, Rodiles A. The fish microbiome and its interactions with mucosal tissues. In: Mucosal Health in Aquaculture. 2015:273-95.

6. Goodrich JK, Waters JL, Poole AC, Sutter JL, Koren O, Blekhman R, Beaumont M, Van Treuren W, Knight R, Bell JT, et al. Human genetics shape the gut microbiome. Cell. 2014:159(4):789-99.

7. Kurilshikov A, Wijmenga C, Fu J, Zhernakova A. Host genetics and gut microbiome: challenges and perspectives. Trends Immunol. 2017;38(9):63347.

8. Spor A, Koren O, Ley R. Unravelling the effects of the environment and host genotype on the gut microbiome. Nat Rev Microbiol. 2011;9(4):279-90.

9. Brown RM, Wiens GD, Salinas I. Analysis of the gut and gill microbiome of resistant and susceptible lines of rainbow trout (Oncorhynchus mykiss). Fish Shellfish Immunol. 2019;86:497-506

10. Egerton S, Culloty S, Whooley J, Stanton C, Ross RP. The gut microbiota of marine fish. Front Microbiol. 2018;9:873.

11. Mohajeri MH, La Fata G, Steinert RE, Weber P. Relationship between the gut microbiome and brain function. Nutr Rev. 2018;76(7):481-96.

12. MichI SC, Ratten JM, Beyer M, Hasler M, LaRoche J, Schulz C. The malleable gut microbiome of juvenile rainbow trout (Oncorhynchus mykiss): dietdependent shifts of bacterial community structures. PLoS One. 2017:12(5): e0177735.

13. Sharp KH, Eam B, Faulkner DJ, Haygood MG. Vertical transmission of diverse microbes in the tropical sponge Corticium sp. Appl Environ Microbiol. 2007; 73(2):622-9.

14. Perez T, Balcazar JL, Ruiz-Zarzuela I, Halaihel N, Vendrell D, de Blas I, Muzquiz $J$. Host-microbiota interactions within the fish intestinal ecosystem. Mucosal Immunol. 2010:3(4):355-60

15. Krajmalnik-Brown R, Ithan ZE, Kang DW, DiBaise JK. Effects of gut microbes on nutrient absorption and energy regulation. Nutr Clin Pract. 2012;27(2): $201-14$

16. Rowland I, Gibson G, Heinken A, Scott K, Swann J, Thiele I, Tuohy K. Gut microbiota functions: metabolism of nutrients and other food components. Eur J Nutr. 2018:57(1):1-24.

17. Panek M, Cipcic Paljetak H, Baresic A, Peric M, Matijasic M, Lojkic I, Vranesic Bender D, Krznaric Z, Verbanac D. Methodology challenges in studying human gut microbiota - effects of collection, storage, DNA extraction and next generation sequencing technologies. Sci Rep. 2018;8(1):5143.

18. Lim MY, Song EJ, Kim SH, Lee J, Nam YD. Comparison of DNA extraction methods for human gut microbial community profiling. Syst Appl Microbiol. 2018:41(2):151-7.

19. Kennedy NA, Walker AW, Berry SH, Duncan SH, Farquarson FM, Louis P, Thomson JM, Consortium UIG, Satsangi J, Flint HJ, et al. The impact of different DNA extraction kits and laboratories upon the assessment of human gut microbiota composition by $16 \mathrm{~S}$ rRNA gene sequencing. PLoS One. 2014;9(2):e88982. 
20. Yuan S, Cohen DB, Ravel J, Abdo Z, Forney LJ. Evaluation of methods for the extraction and purification of DNA from the human microbiome. PLoS One. 2012;7(3):e33865.

21. Tarnecki AM, Rhody NR, Walsh CJ. Health characteristics and blood bacterial assemblages of healthy captive red drum: implications for aquaculture and fish health management. J Aquat Anim Health. 2018:30(4):339-53.

22. Wang AR, Ran C, Ring $\varnothing$, Zhou ZG: Progress in fish gastrointestinal microbiota research. In.: Reviews in Aquaculture; 02 February 2017.

23. Ley RE, Backhed F, Turnbaugh P, Lozupone CA, Knight RD, Gordon J. Obesity alters gut microbial ecology. Proc Natl Acad Sci U S A. 2005;102(31): $11070-5$.

24. Dugas LR, Fuller M, Gilbert J, Layden BT. The obese gut microbiome across the epidemiologic transition. Emerg Themes Epidemiol. 2016;13:2.

25. Mikolajczyk R, Roesner LM. general aspects regarding the skin microbiome. Hautarzt. 2019:70(6):400-6.

26. Semova I, Carten JD, Stombaugh J, Mackey LC, Knight R, Farber SA, Rawls JF. Microbiota regulate intestinal absorption and metabolism of fatty acids in the zebrafish. Cell Host Microbe. 2012;12(3):277-88.

27. Meehan CJ, Beiko RG. A phylogenomic view of ecological specialization in the Lachnospiraceae, a family of digestive tract-associated bacteria. Genome Biol Evol. 2014;6(3):703-13.

28. Liu Y, Li J, Jin Y, Zhao L, Zhao F, Feng J, Li A, Wei Y. Splenectomy leads to amelioration of altered gut microbiota and Metabolome in liver cirrhosis patients. Front Microbiol. 2018;9:963.

29. Neis EP, Dejong $\mathrm{CH}$, Rensen SS. The role of microbial amino acid metabolism in host metabolism. Nutrients. 2015;7(4):2930-46.

30. Liu H, Guo X, Gooneratne R, Lai R, Zeng C, Zhan F, Wang W. The gut microbiome and degradation enzyme activity of wild freshwater fishes influenced by their trophic levels. Sci Rep. 2016;6:24340.

31. White RA, Bjornholt JV, Baird DD, Midtvedt T, Harris JR, Pagano M, Hide W, Rudi K, Moen B, Iszatt N, et al. Novel developmental analyses identify longitudinal patterns of early gut microbiota that affect infant growth. PLoS Comput Biol. 2013;9(5):e1003042.

32. Sharifuzzaman SM, Abbass A, Tinsley JW, Austin B. Subcellular components of probiotics Kocuria SM1 and Rhodococcus SM2 induce protective immunity in rainbow trout (Oncorhynchus mykiss, Walbaum) against Vibrio anguillarum. Fish Shellfish Immunol. 2011;30(1):347-53.

33. Lebeer S, Vanderleyden J, De Keersmaecker SC: Genes and molecules of lactobacilli supporting probiotic action. Microbiol Mol Biol Rev 2008, 72(4): 728-764, Table of Contents.

34. Seo B, Yoo JE, Lee YM, Ko G. Sellimonas intestinalis gen. Nov., sp. nov., isolated from human faeces. Int J Syst Evol Microbiol. 2016;66(2):951-6.

35. Rolhion N, Chassaing B. When pathogenic bacteria meet the intestinal microbiota. Philos Trans R Soc Lond Ser B Biol Sci. 2016;371(1707).

36. Kandi V, Palange $P$, Vaish $R$, Bhatti $A B$, Kale V, Kandi MR, Bhoomagiri MR. Emerging bacterial infection: identification and clinical significance of Kocuria species. Cureus. 2016;8(8):e731.

37. Yang I, Corwin EJ, Brennan PA, Jordan S, Murphy JR, Dunlop A. The infant microbiome: implications for infant health and neurocognitive development. Nurs Res. 2016;65(1):76-88.

38. Larsen AM, Mohammed HH, Arias CR. Characterization of the gut microbiota of three commercially valuable warmwater fish species. J Appl Microbiol. 2014;116(6):1396-404.

39. Leeds TD, Silverstein JT, Weber GM, Vallejo RL, Palti Y, Rexroad CE 3rd Evenhuis J, Hadidi S, Welch TJ, Wiens GD. Response to selection for bacterial cold water disease resistance in rainbow trout. J Anim Sci. 2010;88(6):1936-46.

40. Sambrook J, Russell DW. Purification of nucleic acids by extraction with phenol:chloroform. CSH Protoc. 2006;2006(1)

41. Parada AE, Needham DM, Fuhrman JA. Every base matters: assessing small subunit rRNA primers for marine microbiomes with mock communities, time series and global field samples. Environ Microbiol. 2016;18(5):1403-14

42. ILLUMINA: Miseq System Guide. In., vol. Document \# 1000000061014 v00 2018

43. Schloss PD, Westcott SL, Ryabin T, Hall JR, Hartmann M, Hollister EB, Lesniewski RA, Oakley BB, Parks DH, Robinson CJ, et al. Introducing mothur: open-source, platform-independent, community-supported software for describing and comparing microbial communities. Appl Environ Microbiol. 2009;75(23):7537-41.

44. Kozich JJ, Westcott SL, Baxter NT, Highlander SK, Schloss PD. Development of a dual-index sequencing strategy and curation pipeline for analyzing amplicon sequence data on the MiSeq Illumina sequencing platform. Appl Environ Microbiol. 2013;79(17):5112-20.

45. Carlsen $T$, Aas $A B$, Linder $D$, al. e: Don't make a mista(g)ke: is tag switching an overlooked source of error in amplicon pyrosequencing studies? . In., vol. 5: Fungal Ecol; 2012: 747-749.

46. Rognes T, Flouri T, Nichols B, Quince C, Mahe F. VSEARCH: a versatile open source tool for metagenomics. PeerJ. 2016;4:e2584.

47. Lindahl BD, Nilsson RH, Tedersoo L, Abarenkov K, Carlsen T, Kjoller R, Koljalg U, Pennanen T, Rosendahl S, Stenlid J, et al. Fungal community analysis by high-throughput sequencing of amplified markers--a user's guide. New Phytol. 2013:199(1):288-99.

48. Walker DM, Leys JE, Grisnik M, Grajal-Puche A, Murray CM, Allender MC. Variability in snake skin microbial assemblages across spatial scales and disease states. ISME J. 2019;13(9):2209-22.

49. Walker DM, Murray CM, Talbert D, Tinker P, Graham SP, Crowther TW. A salamander's top down effect on fungal communities in a detritivore ecosystem. FEMS Microbiol Ecol. 2018;94(12).

\section{Publisher's Note}

Springer Nature remains neutral with regard to jurisdictional claims in published maps and institutional affiliations.
Ready to submit your research? Choose BMC and benefit from:

- fast, convenient online submission

- thorough peer review by experienced researchers in your field

- rapid publication on acceptance

- support for research data, including large and complex data types

- gold Open Access which fosters wider collaboration and increased citations

- maximum visibility for your research: over $100 \mathrm{M}$ website views per year

At $\mathrm{BMC}$, research is always in progress.

Learn more biomedcentral.com/submissions 